\title{
Tangence
}

\section{La constellation des filles : du script romantique à la communauté solidaire dans Le goudron et les plumes d'Hélène Monette}

\section{The constellation of girls: from the romantic script to the community of solidarity in Hélène Monette's Le goudron et les plumes}

\section{Catherine Dussault Frenette}

Numéro 119, 2019

« Fille-s » dans l’imaginaire littéraire au Québec (1980-2015)

URI : https://id.erudit.org/iderudit/1065671ar

DOI : https://doi.org/10.7202/1065671ar

Aller au sommaire du numéro

Éditeur(s)

Tangence

ISSN

1189-4563 (imprimé)

1710-0305 (numérique)

Découvrir la revue

Citer cet article

Dussault Frenette, C. (2019). La constellation des filles : du script romantique à la communauté solidaire dans Le goudron et les plumes d'Hélène Monette.

Tangence, (119), 113-134. https://doi.org/10.7202/1065671ar

\section{Résumé de l'article}

La figure de la fille en tant qu'amie a, jusqu'à tout récemment, été peu abordée par les études littéraires. Cet article propose une réflexion sur les amitiés entre filles, telles qu'elles sont dépeintes dans le premier roman d'Hélène Monette, Le goudron et les plumes, paru en 1993. En faisant s'entrecroiser les voix de deux narratrices autrefois amies, l'écrivaine retrace la généalogie de cette amitié brisée, tout en levant le voile sur le système qui, en amont, entraîne les filles à se positionner comme rivales. L'analyse présentée s'attarde tout d'abord à relever la critique qu'adresse l'oeuvre au script romantique hétéronormatif, qui repose sur l'élection, par un homme, d'une seule fille du groupe, entraînant ainsi la désolidarisation de celles qui le composent. Sont ensuite exposées les diverses voies de sortie dessinées par Monette, hors des schémas patriarcaux, qui valorisent les relations hétérosexuelles exclusives au détriment des amitiés féminines, et vers une nouvelle économie des relations entre filles, fondées sur une reconnaissance réciproque, dont les termes sont définis au sein même du groupe des semblables. La lecture présentée suggère que l'investissement de ce pouvoir passe avant tout par l'élaboration d'un nouveau symbolisme au féminin, représenté, dans l'oeuvre, par la métaphore de la constellation. 
Tangence $\square \mathrm{n}^{\circ}$ 119, 2019, p. 113-134.

\title{
La constellation des filles: du script romantique à la communauté solidaire dans Le goudron et les plumes d'Hélène Monette \\ Catherine Dussault Frenette \\ Université de Sherbrooke
}

\begin{abstract}
Ne plus être obligées d'adorer Zeus tout en se faisant fourrer. Ne plus être la fille dans le sac à vidanges, qu'on entend encore hurler, du dépotoir des sentiments jusqu'au centre-ville. Ne plus être la salope de quelqu'un, la chiante de tout le monde, l'abjecte des salauds. Oh boy! La santé! On retrouverait le goût d'avoir du plaisir et de rire comme des folles.
\end{abstract}

Hélène Monette ${ }^{1}$

Tout commence par l'exergue: celui choisi par Hélène Monette pour Le goudron et les plumes (1993) est tiré du Ravissement de Lol V. Stein de Marguerite Duras (1964), dans lequel Tatiana Karl annonce à Lol qu'elle «ne sai[t] pas si [elle la] reverr[a] aussi souvent que [Lol a] l'air de le souhaiter ${ }^{2}$ ». Car elle a «des amants» qui «occupent [s] on temps libre complètement ${ }^{3} »$. Qui plus est, la Tatiana de Duras laisse entendre que ce n'est pas par la force aléatoire des choses

1. Hélène Monette, Le goudron et les plumes [1993], Montréal, Boréal, coll. «Boréal compact», 2001, p. 155. Désormais, les références à cet ouvrage seront indiquées par le sigle GP, suivi de la page, et placées entre parenthèses dans le corps du texte.

2. Marguerite Duras, Le ravissement de Lol V. Stein [1964], Paris, Gallimard, coll. «Folio», 1982, p. 96.

3. Marguerite Duras, Le ravissement de Lol V. Stein, ouvr. cité, p. 97. 
qu'elle consacrera davantage de temps à ses amants qu'à son amie. Au contraire, elle insiste: "Je désire que ce soit ainsi », déclare-t-elle à une Lol «trist $[\mathrm{e}]$ » et «découragée » ${ }^{4}$. Voilà précisément ce que Monette entreprend d'explorer, voire de démystifier dans son premier roman, soit cet octroi d'une valeur supérieure aux relations hétérosexuelles, qui se mesure ici au temps attribué aux amants (masculins), et ce, au détriment des amitiés entre filles. L'écrivaine propose, en cela, une déconstruction de ce que Kathleen Barry a appelé «l'identification masculine», c'est-à-dire «l'acte par lequel les femmes ${ }^{5}$ placent les hommes au-dessus des femmes, elles-mêmes y comprises", faisant en sorte que "[1]'interaction avec les femmes [soit] vue comme une forme inférieure de relation à tous les niveaux ${ }^{6} »$. C'est également ce que soulignait bell hooks, dans son article «Sisterhood: Political Solidarity between Women»: «L'idéologie suprémaciste mâle encourage les femmes à croire que nous sommes sans valeur et que nous n'obtenons de valeur qu'en entrant en relation avec des hommes. On nous enseigne que les relations que nous entretenons les unes avec les autres diminuent notre expérience, plutôt que de l'enrichir ${ }^{7}$.» En opérant, par le biais de ses deux narratrices féminines, une critique du script romantique hétéronormatif, Monette lève le voile sur ce processus par lequel les femmes délaissent leurs sœurs pour "se mettre dans le camp des hommes ${ }^{8}$ », exposant, par le fait même, le fonctionnement d'un système qui fait du renoncement aux unes la condition du désir vers l'autre.

Le goudron et les plumes met en scène un dialogue différé entre Cora Corail et Léa, devenues rapidement amies lors $d^{\prime}$ ' $u$ une nuit de mars» $(G P$, p. 21) particulièrement mouvementée, marquée par la consommation de cocaïne dans les toilettes d'un bar miteux

4. Marguerite Duras, Le ravissement de Lol V. Stein, ouvr. cité, p. 97.

5. Si Barry utilise le terme "femmes», je privilégierai, pour ma part, celui de «filles», puisque c'est ainsi que se désignent les narratrices de Monette.

6. Kathleen Barry, Female Sexual Slavery [1979], citée par Adrienne Rich, «La contrainte à l'hétérosexualité et l'existence lesbienne ", Nouvelles Questions féministes, $\mathrm{n}^{\circ} 1,1981, \mathrm{p} .29-30$.

7. «Male supremacist ideology encourages women to believe we are valueless and obtain value only by relating to or bonding with men. We are taught that our relationships with one another diminish rather than enrich our experience." bell hooks, "Sisterhood: Political Solidarity between Women », Feminist Review, $\mathrm{n}^{\circ} 23,1986$, p. 127 ; je traduis.

8. Kathleen Barry, citée par Adrienne Rich, «La contrainte à l'hétérosexualité et l'existence lesbienne», art. cité, p. 29. 
de Montréal et la fuite dans les rues de la ville pour échapper à la colère d'un chauffeur de taxi enragé par l'insouciance et la bonne humeur des filles qui lui demandent, à la blague, de les emmener « [e]n Espagne» $(G P$, p. 27) — signe de la résistance, parfois violente, des hommes à la complicité des filles, j'y reviendrai ${ }^{9}$. Dès les premiers instants de cette rencontre inattendue, les protagonistes sont déjà «complices», «déjà liées» (GP, p. 24). Quelque chose, pourtant, semble avoir mis fin à leur amitié: aux yeux de Léa, à qui Cora Corail fait parvenir quatre cassettes sur lesquelles elle s'adresse à l'amie perdue, ces enregistrements représentent «une sorte de testament de [leur] amitié» (GP, p. 20). Par la voix de ces deux personnages féminins dont les discours alternent d'un chapitre à l'autre, Monette recompose la généalogie d'une amitié brisée (qui, comme le texte l'indique bien, est une parmi tant d'autres) par les contraintes imposées par les relations hétérosexuelles exclusives que les filles vivent l'une et l'autre, chacune de son côté. Le récit, toutefois, va bien audelà du deuil occasionné par la rupture amicale; le discours attribué au personnage de Cora Corail donne à voir l'éveil d'une «conscience de genre» laquelle, selon Eleni Varikas, se caractérise par une «prise de conscience par les femmes de leur existence sociale» et par une étape dans «le long processus qui aboutit à la formation d'une conscience féministe ${ }^{10} »$. Dans Le goudron et les plumes, cette prise de conscience, d'abord individuelle, puis collective, du statut minorisé des filles, qui permet de passer de la sphère intime à l'action politique, s'opère en trois temps: d'abord, par la dénonciation du script romantique, abondamment relayé par les contes de fées, qui repose sur l'élection, par un homme (un prince), d'une seule fille du groupe (la princesse), entraînant ainsi la désolidarisation des filles qui le composent; ensuite, par le retrait volontaire, de la part de

9. Selon Françoise Collin, la division des femmes est notamment assurée par la structure familiale patriarcale «contrôlée [...] par les hommes qui perçoivent comme une menace tout rassemblement, toute réunion de femmes où ils ne sont pas admis, qu'ils ne contrôlent pas. Au point qu'aujourd'hui la moindre rencontre est suspectée d'intentions incendiaires et prend figure de "complot".» ( No man's land: réflexion sur l'esclavage volontaire des femmes», dans Maria A. Macciochi [dir.], Les femmes et leurs maîtres, Paris, Christian Bourgois éditeur, 1978, p. 270.) Désormais, les références à cet article seront indiquées par le sigle $N M L$, suivi de la page, et placées entre parenthèses dans le corps du texte.

10. Eleni Varikas, La révolte des dames. Genèse d'une conscience féministe dans la Grèce du XIX $x^{e}$ siècle (1833-1908), thèse de doctorat, Université Paris-VII, 1986, p. 10-11 et 13-14. 
Cora Corail, de la mire du male gaze ${ }^{11}$, lequel passe par l'abandon du masque de la féminité normative ${ }^{12}$. Enfin, cette sortie hors du régime hétéronormatif rend possible la création d'un nouveau modèle de relations entre filles, reposant sur la réciprocité. À cet égard, Monette dissémine, à travers les discours entrelacés de Cora Corail et de Léa, les valeurs privilégiées à partir desquelles fonder un nouveau symbolisme entre filles, qui, comme l'écrivait Luce Irigaray, représente un «intervalle d'échange, ou de paroles, ou de gestes ${ }^{13}$ » permettant ultimement de remplacer la rivalité entre filles, indispensable au maintien du pouvoir patriarcal, par un amour réciproque ${ }^{14}$. Dans le roman de Monette, ce symbolisme s'incarne dans une image précise: celle de la constellation (GP, p. 54) qui, dans son sens propre, représente un regroupement d'étoiles, reliées entre elles pour former une figure mythologique. En en faisant un usage métaphorique, l'écrivaine semble appeler à la création d'un nouveau régime, élaboré cette fois par et pour les filles, lesquelles ont, de tout temps, été exclues à titre de sujets de la composition des grands récits sur lesquels reposent nos sociétés occidentales.

Le premier roman d'Hélène Monette offre un terrain d'analyse des plus riches pour aborder l'une des facettes encore peu étudiées de la figure de la fille dans la littérature québécoise, soit celle de l'amie ou de l'alliée ${ }^{15}$. Mais, avant de me pencher précisément sur

11. Voir Laura Mulvey, Visual Pleasure and the Narrative Cinema [1975], Houndmills, Basingstoke [Hampshire, England]/New York, Palgrave Macmillan, 2009.

12. Voir Joan Riviere, "Womanliness as a Masquerade », The International Journal of Psychoanalysis, $\mathrm{n}^{\circ} 10,1929, \mathrm{p} .303-313$; Luce Irigaray, Ce sexe qui n'en est pas un, Paris, Minuit, coll. "Critique», 1977 et Judith Butler, Trouble dans le genre. Le féminisme et la subversion de l'identité [1990], trad. de l'anglais par Cynthia Kraus, Paris, La Découverte, 2006.

13. Luce Irigaray, Éthique de la différence sexuelle, Paris, Minuit, coll. "Critique», 1984, p. 103; l'autrice souligne.

14. Cet amour qui, selon Martine Delvaux, n'a rien à voir avec «cet amour rose bonbon qu'on cherche tant à associer aux femmes comme s'il leur était naturel, ou à cet amour pseudo-mystique qui demeure trop souvent une façon d'échapper au politique», mais qui renvoie plutôt à "l'“amour" au sens d'alliance, comme la force d'attraction qui fait que les filles peuvent être ensemble sans en mourir.» (Les filles en série. Des Barbies aux Pussy Riot, Montréal, Les éditions du remueménage, 2013, p. 217.) Voir aussi Luce Irigaray, Éthique de la différence sexuelle, ouvr. cité, p. 103.

15. Parmi les études ayant été réalisées sur la question, notons, parmi quelques autres, l'article de Lori Saint-Martin, "L'amitié, c'est mieux que la famille". Rapports amicaux entre femmes dans le roman québécois», Nouvelles questions féministes, vol. 30, n² 2, 2011, p. 76-91 et celui d'Isabelle Boisclair, «La solidarité 
ce que le rapport de cette figure aux autres filles nous apprend tant sur l'imaginaire qui la fonde que sur ses potentialités subversives ${ }^{16}$, il est nécessaire d'effectuer un détour par sa relation avec les hommes - trajectoire qu'emprunte également l'œuvre de Monette. Car c'est bien le rapport aux mâles qui prime dans le processus de socialisation des filles ${ }^{17}$, les hommes occupant de fait, en contexte patriarcal, la position du sujet universel, la position de l'Un, par rapport auquel sont contraintes de se définir les Autres ${ }^{18}$. Alors que la «fille» a longtemps été pensée en fonction de son lien avec les hommes, qui la définit comme objet approprié ${ }^{19}$, il est temps de réfléchir au potentiel de résistance que recèle cette figure - comme l'a bien montré Martine Delvaux ${ }^{20}$, entre autres —, potentiel qui se déploie, le plus souvent, grâce à la reconnaissance octroyée par le groupe des semblables, à travers les relations d'amitié.

Ma lecture de l'œuvre de Monette sera notamment nourrie par les réflexions proposées par Françoise Collin sur la prétendue " complicité» des filles et des femmes à leur propre oppression dans son article «No man's land: réflexion sur "l'esclavage volontaire" des femmes». Aussi, puisque je m'intéresserai à l'identité «fille», et que l'identité est toujours façonnée par les interactions avec les autres, je prendrai appui sur la théorie de l'intersubjectivité, laquelle, selon la psychanalyste féministe Jessica Benjamin, «considère [l'] unité [du moi] comme un point particulier dans l'éventail des relations, et non pas comme "l'état de nature", originel de l'indi$v^{2} \mathrm{vu}^{21}$ ». La notion de «mascarade», proposée par Luce Irigaray, qui renvoie au devoir imposé aux filles et aux femmes de refléter

féminine comme réponse à la domination masculine: étude de deux motifs genrés dans l'œuvre d'Anne Hébert », Les Cahiers Anne-Hébert, nº 8, 2008, p. 15-36.

16. Voir Élaine Audet, Le cour pensant. Courtepointe de l'amitié entre femmes, Québec, Le Loup de Gouttière, 2000, p. 12.

17. Voir Kathleen Barry, citée dans Adrienne Rich, «La contrainte à l'hétérosexualité et l'existence lesbienne», art. cité; Janet Holland, Caroline Ramazanoglu, Sue Sharpe et Rachel Thomson, The Male in the Head. Young People, Heterosexuality and Power [1998], London, The Tufnell Press, 2004 et Monique Wittig, La pensée straight [2001], Paris, Éditions Amsterdam, 2013.

18. Voir Simone de Beauvoir, Le deuxième sexe, Paris, Gallimard, coll. «Folio », 1949, t. I, «Les faits et les mythes».

19. Voir Colette Guillaumin, «Pratique du pouvoir et idée de Nature (1). L'appropriation des femmes », Questions féministes, no 2, 1978, p. 5-30.

20. Martine Delvaux, Les filles en série. Des Barbies aux Pussy Riot, ouvr. cité.

21. Jessica Benjamin, Les liens de l'amour [1988], trad. de l'anglais par Madeleine Rivière, Paris, Métailié, 1992, p. 26. 
les fantasmes masculins, sera également centrale à l'analyse, dans la mesure où le renoncement, par Cora Corail, à la féminité normative constitue un point tournant de l'œuvre à partir duquel le personnage féminin passe de la docilité à la résistance. Enfin, la réflexion proposée dans les pages qui suivent est alimentée par les travaux des chercheuses s'étant intéressées aux amitiés féminines, tant d'un point de vue sociologique que littéraire, dont bell hooks ${ }^{22}$, Élaine Audet $^{23}$ et Lori Saint-Martin ${ }^{24}$.

\section{Le script romantique et la désolidarisation des filles}

Dans son article phare sur l'hétéronormativité et l'existence lesbienne, Adrienne Rich envisage "l'institution de l'hétérosexualité [...] comme tête de pont de la domination masculine ${ }^{25} »$. Or, l'une des stratégies déployées par le dispositif ${ }^{26}$ hétéronormatif, qui assure la constante reproduction de ce dernier, est sans conteste l'idéalisation de l'amour romantique, tel que la véhiculent «les contes de fées, la télévision, les films, la publicité, l'imagerie du "mariage-enblanc"27». L'impact des réactualisations de ce modèle relationnel sur l'imaginaire s'avère d'autant plus important que celles-ci reconduisent toujours le même script — à quelques variations près —, savamment articulé et reconnaissable entre tous puisqu'il s'agit, bien

22. bell hooks, «Sisterhood: Political Solidarity between Women», art. cité.

23. Élaine Audet, Le cour pensant. Courtepointe de l'amitié entre femmes, ouvr. cité.

24. Lori Saint-Martin, "L'amitié, c'est mieux que la famille"», art. cité. Analysant les «tensions narratives» entre les rapports hétérosexuels et les relations d'amitié entre femmes chez six écrivaines québécoises (Laure Conan, Germaine Guèvremont, Gabrielle Roy, Anne Hébert, Francine Noël et Nelly Arcan), l'autrice constate certaines «limites que pose à l'amitié entre femmes l'économie hétérosexuelle.» Malgré cela, il semble que «l'amitié entre femmes apporte aux personnages consolation, force de résistance, ou plaisirs intimes et quotidiens selon le cas ", ce qu'il est également possible d'observer dans l'œuvre de Monette étudiée ici (p. 77 et 90).

25. Adrienne Rich, «La contrainte à l'hétérosexualité et l'existence lesbienne», art. cité, p. 17.

26. Selon Giorgio Agamben, lequel s'inspire des travaux de Foucault sur la question, un dispositif représente «tout ce qui a, d'une manière ou d'une autre, la capacité de capturer, d'orienter, de déterminer, d'intercepter, de modeler, de contrôler et d'assurer les gestes, les conduites, les opinions et les discours des êtres vivants.» Il s'agit, en somme, d' « une machine qui produit des subjectivations» (Qu'est-ce qu'un dispositif? [2006], Paris, Rivage poche, coll. «Petite Bibliothèque», 2014, p. 31 et 42 ).

27. Adrienne Rich, «La contrainte à l'hétérosexualité et l'existence lesbienne», art. cité, p. 28-29. 
souvent, du premier du genre à être imposé (surtout) aux jeunes filles, par l'entremise des contes de fées. Selon Bertrand Gervais, lequel s'appuie sur les définitions proposées par Schank et Abelson, «le script permet de décrire des actions relativement complexes [...] c'est-à-dire des séquences prédéterminées et stéréotypées d'actions définissant des situations communes». Il s'agit, en sémiotique, d'une «structure événementielle à forte régularité ${ }^{28}$ ».

Dans les enregistrements qu'elle fait parvenir à Léa, Cora Corail s'emploie à revisiter leur "genèse affective» $(G P$, p. 152) et désigne, ce faisant, le script romantique traditionnel comme responsable de la fin de l'amitié entre les deux filles. C'est d'abord le caractère fondamentalement mortifère de ce script que déplore la narratrice, qui livre, de son propre aveu, un «combat déroutant [...] aux contes de fées dont [elle] semble [paradoxalement] ne pas pouvoir se passer» (GP, p. 82; l'ajout entre crochets est de moi). Les différents noms dont elle l'affuble en témoigne: aux yeux de Cora Corail, le romantisme hétérosexuel est un «miroir brisé», un «cauchemar éternel» $(G P$, p. 81$)$, « un beau petit martyre» (GP, p. 79), un « conte barbare» (GP, p. 106) qui fonctionne grâce à des «philtres maudits» (GP, p. 113). D'autres dénominations traduisent l'influence de la dimension économique dans l'ordonnancement des positions féminine et masculine au sein du système hétéronormatif ${ }^{29}$; le script romantique devient, par la voix de Cora Corail, «la passion selon l'industrie» $(G P$, p. 87) ou encore «le marché de l'amour» (GP, p. 111). Les noms donnés à ceux qui tirent profit de cet arrangement des sexes soulignent, quant à eux, la violence intrinsèque du script. Dans le monde de la narratrice, la communauté des hommes n'a pas bonne

28. Bertrand Gervais, Récits et actions. Pour une théorie de la lecture, Longueuil, Le Préambule, coll. "L’Univers des discours», 1990, p. 164-165 et 226. Sur le script hétéronormatif reconduit par les contes de fées traditionnels, voir Jennifer Waelti-Walters, Fairy Tales and the Female Imagination, Montréal, Eden Press, 1982.

29. À ce sujet, voir la notion d'échange économico-sexuel de Paola Tabet selon laquelle il y a, «[d] ans une écrasante majorité [des] relations [...], de la part des femmes, $[\ldots]$ fourniture d'un service ou d'une prestation, variable en nature et en durée, mais comprenant l'usage sexuel ou se référant à la sexualité [et], de la part des hommes, il y a remise d'une compensation ou rétribution d'importance et de nature variables, mais de toute façon liée à la possibilité d'usage sexuel de la femme, à son accessibilité sexuelle.» (La grande arnaque. Sexualité des fermmes et échange économico-sexuel, trad. de l'italien par Josée Contréras, Paris, L'Harmattan, coll. «Bibliothèque du féminisme», 2004, p. 8.) 
presse. Elle évoque tour à tour « [1] es barbares de l'entrecuisse» (GP, p. 41) qui ont croisé son chemin et « tous ces butés qui [1]'ont sautée avec l'âme d'un revolver» (GP, p. 118).

Comment expliquer, alors, que les filles continuent de jouer leur rôle dans cette mise en scène qui les désavantage et les fait souffrir? C'est qu'elles ont, comme le suggère Monette à travers le discours de Cora Corail, l'imaginaire colonisé ${ }^{30}$, voire «anesthési [é $]^{31}$ » par la constante répétition du Même, qui laisse des «[t]races insidieuses dans le paysage du cœur» (GP, p. 153):

Je crois que les filles, Nastassja ${ }^{32}$, adorent les hommes mais on leur a dit, depuis toujours, dans les films comme sur le champ de bataille, qu'ils faisaient souffrir. Alors. Bouche tes oreilles, Louisianna: les filles souffrent par anticipation et par conditionnement. C'est ce qu'elles ont appris dans la confusion des secousses filmiques et des tremblements de rage. [...] Labyrinthe à panique, souricière bloquée par les ruines de l'amertume. Voilà la scène. (GP, p. 151)

Selon Cora Corail, les filles souffrent car on ne leur a appris qu'à attendre: leur "éducation viciée de romantisme à la Doris Day» ( $G P$, p. 153) a supprimé, à leurs yeux, la possibilité même de se faire sujet et d'agir en fonction de leurs désirs:

On nous a appris à attendre! Tu vois, il y a des filles qui portent la camisole de force à cause de ça. Psychose de l'attente. Personne n'est jamais arrivé. Personne n’a jamais été tendre. [...] On ne les

30. La notion de «colonisation de l'imaginaire» est employée par Katherine Roussos dans son ouvrage Décoloniser l'imaginaire. Du réalisme magique chez Maryse Condé, Sylvie Germain et Marie Ndiaye. Prenant appui sur les travaux de divers penseur·euse's de l'approche décoloniale, dont Frantz Fanon, Edward Saïd et Audre Lorde, la chercheuse attire l'attention, avec cette notion, sur le pouvoir que possèdent les «images et les valeurs» du groupe dominant d'orienter et de façonner l'imaginaire des sujets se trouvant en situation de «colonisé(e)» (Katherine Roussos, Décoloniser l'imaginaire. Du réalisme magique chez Maryse Condé, Sylvie Germain et Marie Ndiaye, Paris, L’Harmattan, coll. «Bibliothèque du féminisme», 2007, p. 8).

31. Nicole-Claude Mathieu, "Quand céder n'est pas consentir» [1985], dans L'anatomie politique. Catégorisations et idéologies du sexe, Donnemarie-Dontilly, Éditions iXe, 2013, p. 199.

32. Cora Corail s'adresse à Léa en lui donnant chaque fois un nom différent. Je reviendrai sur cet aspect particulier de l'œuvre, de même que sur la signification qu'on peut lui prêter. 
avait pas averties, non plus, de s'aimer, de se donner la vie, et toute une: la leur, sapristi!

Le désastre est entier. Les filles ne savent pas aimer. Elles sont trop coincées par l'attente, donc la peur, le manque de confiance, la douleur sans cesse anticipée. Elles aime[nt] mieux jouer dans un film, attendre les fleurs, le coup de fil. (GP, p. 153)

Sans pouvoir, chacune de celles qui compose le groupe des filles se résigne à chercher la reconnaissance de sa propre existence dans le regard d'un homme qui, un beau jour, l'élira parmi ses semblables et l'intègrera à son monde - celui des hommes, en l'occurrence. Cela va de pair avec ce que Françoise Collin appelle le «mythe de l'Unique ${ }^{33} »$, lequel engendre la désolidarisation des filles à l'égard de l'idée d'incarner cette «élue ${ }^{34} »$ : «La culture occidentale tout au moins veille à donner à chaque femme l'impression qu'elle est unique en son genre, et la pousse à défendre cette unicité contre les autres, vécues comme menaçantes. Chacune est une, élue entre toutes» (NML, p. 270). Le script romantique fait ainsi se retourner les filles les unes contre les autres ${ }^{35}$; il se présente, en cela, comme un dispositif servant à fabriquer des rivales, comme le laisse entendre Cora Corail:

Si j'aime, elles s'imaginent que je suis aimée. Si j'aime, elles se sentent retournées dans leur attente pesante, déjouées par leur solitude encombrante. Et quand je suis aimée, c'est pire. Elles me crachent au visage, sortent le dossier noir de l'élu, me font de la peine. Quand je suis aimée, elles n'en reviennent pas de ne

33. Ce que confirme le discours que tient Cora Corail à propos du sentiment d'élection: «Les filles sont butées et terriblement égocentriques. Elles croient toujours que c'est exclusivement pour elles, très uniques détentrices du grand amour» (GP, p. 79-80).

34. Luce Irigaray abonde en ce sens: «[Les femmes] sont à la fois complices et rivales pour advenir à l'unique position possible dans le désir de l'homme. Cette compétition paralyse aussi l'amour entre femmes-sœurs. Et parce qu'elles travaillent à obtenir le poste de l'unique» (Éthique de la différence sexuelle, ouvr. cité, p. 101 ; l'autrice souligne).

35. Si le script romantique représente, dans l'œuvre de Monette, le principal responsable de la désolidarisation des filles, il faut bien voir que le sexisme qui sous-tend celui-ci n'est qu'un des multiples aspects de la culture hétéropatriarcale qui divise les femmes. Comme le soulignait bell hooks, «les femmes sont divisées par les attitudes sexistes, le racisme, le privilège de classe et une foule d'autres préjugés.» («Sisterhood: Political Solidarity between Women», art. cité, p. 127; je traduis. "[w] $[$ omen are divided by sexist attitudes, racism, class privilege, and a host of other prejudices.») 
pas l'être, ça devient une obsession: elles cassent tout. Elles n'ont aucune empathie pour les saveurs tendres que je peux m'offrir. Jalouses. Jalouseries. (GP, p. 142)

Or ce qu'illustre le discours de la narratrice, c'est que cette voie, qui est celle de la rivalité, est sans issue pour le sujet féminin qui s'y engage. Car nier sa semblable, c'est aussi, un peu, se nier soi-même. Dans l'œuvre de Monette, les filles déçues par l'amour romantique (celles que la narratrice appelle «les princesses esseulées»; GP, p. 151) se laissent dépérir. «[L] es femelles de la terre [...] se donnent un ventre creux en période cruelle» (GP, p. 158), déclare Cora Corail en parlant des filles mais, surtout, en parlant d'elle-même ( «Je n'ai jamais parlé que pour moi» [GP, p. 154], précise-t-elle à Léa, indiquant, par ce passage constant du collectif à l'individuel et vice-versa, que cette condition est partagée et que de cette expérience commune de l'oppression ${ }^{36}$ peut, éventuellement, naître la révolte). Plus encore, la restriction des possibles de l'amour au seul script romantique contraint les filles à s'accommoder de leur souffrance ${ }^{37}$, si ce n'est de s'en réjouir comme d'une chance inespérée:

Lorsque tout se brise, les illusions, le rêve, et cette espèce de joie puérile qui simulait les forces vives du plus parfait abandon, elles n'ont plus l'air que d'un chien, et une par une, elles n'ont plus l'air de rien, elles n'ont plus qu'une chienne de vie à se mettre sous la dent dure, belles aux joues creuses, le corps patraque. Elles sont alors piteuses, si tu voyais ça. Presque heureuses d'avoir connu l'erreur encore une fois, presque rassurées qu'ils les aient prises malgré tout dans leurs bras, tout à fait masos de se répéter, inlassablement: je ne l'aime plus, il ne m'aimait pas. (GP, p. 80)

Ainsi se répète sans relâche l'histoire des filles, prisonnières de la «fixité répétitive» (NML, p. 273) du script imposé, du moins jusqu’à ce que l'une d'entre elles dévoile «le mensonge, le leurre» $(G P, \mathrm{p} .79)$ que sont les contes de fées, ces «ragots sentimentaux» (GP, p. 153) destinés à « tout étourdir, même la conscience d'une fille intelligente »

36. Lori Saint-Martin, Contre-voix. Essais de critique au féminin, Montréal, Nuit Blanche, 1997, p. 25-26.

37. Sur la question des contraintes posées à l'imaginaire, voir Catherine Dussault Frenette, La fabrique du désir féminin. Le dispositif de la contrainte dans la littérature contemporaine des femmes (1990-2015), thèse de doctorat, Université de Sherbrooke, 2018. 
(GP, p. 79), mettant ainsi à nu le processus par lequel le désir d'être reconnue emprunte la voie du masochisme ${ }^{38}$.

\section{Retirer le masque de la féminité normative}

Nous l'avons vu, le script romantique commande à chaque fille de se constituer en tant qu'Unique. Or, «[p]aradoxalement, chacune ne peut être unique que si elle est toutes, si elle réalise l'idée de la Féminité, définie par quelques composantes élémentaires» (NML, p. 271). Les femmes, selon Luce Irigaray, n'ont d'autre choix, pour s'inscrire à leur tour dans l'économie du désir, que de porter un masque - lequel est à la fois artifice (maquillage, chirurgies esthétiques) et performance (postures, attitudes), cela rappelant la «burqa de chair» de Nelly Arcan ${ }^{39}$. La mascarade permet à cellesci de «récupérer quelque chose du désir, pour participer au désir de l'homme, mais au prix de renoncer au leur. Dans la mascarade, elles se soumettent à l'économie dominante du désir, pour essayer de rester quand même sur le "marché". Mais c'est du côté de ce dont on jouit et non de qui jouit ${ }^{40}{ }$. Dans l'œuvre de Monette, Cora Corail expérimente successivement les deux options offertes aux filles dans le régime hétéronormatif, la première étant de revêtir le masque — et de demeurer, ce faisant, du côté de l'objet (être celle «dont on jouit») — la seconde étant de le refuser ou, dans ce cas-ci, de le retirer, au risque de disparaître. Car, dans la mesure où les hommes sont les seuls sujets du script romantique, et que seul un sujet est apte à reconnaître, celle qui ôte le masque se trouve privée de la reconnaissance essentielle à toute affirmation de soi $^{41}$.

Au moment où la narratrice entame son premier enregistrement, le masque est déjà tombé. Ce n'est pas faute, toutefois, d'avoir essayé de le faire tenir en place. Faisant le récit, à Léa, d'une soirée passée chez elle en compagnie d'un homme ${ }^{42}$, elle lui fait part de la

38. Voir Jessica Benjamin, Les liens de l'amour, ouvr. cité, p. 121-127.

39. La «burqa de chair» est une expression forgée par Nelly Arcan dans À ciel ouvert. La burqa de chair renvoie au corps féminin modelé par l'" acharnement esthétique» (À ciel ouvert, Paris, Seuil, 2007, p. 121), de façon à correspondre aux diktats de beauté définis par le regard masculin désirant.

40. Luce Irigaray, Ce sexe qui n'en est pas un, ouvr. cité, p. 131-132.

41. Voir Jessica Benjamin, Les liens de l’amour, ouvr. cité, p. 27.

42. Du propre aveu de Cora Corail, cette rencontre, à laquelle elle fait allusion, n'a pas réellement eu lieu. Mais le récit qu'elle en fait respecte néanmoins, en tout point, ce qui est déjà arrivé et continuera d'arriver: «Il n'est même pas venu, j'ai tout inventé. J'ai lu dans le futur ce qui s'est toujours passé. Je t'ai tout de même 
façon dont elle prévoyait se présenter à ce dernier: «j'allongerai les bras, poserai mes mains sur ses épaules, genoux frissonnants contre ses cuisses entrouvertes. Je lui dirai: regarde celle que j'ai inventée, c'est moi.» (GP, p. 85) Se fondre dans le masque, l'investir comme une seconde peau: voilà la tentative désespérée des filles de Monette pour se faire aimer des «héros délicats» $(G P$, p. 79), figures relevant elles aussi du mythe, selon Cora Corail ${ }^{43}$. Cette entreprise échoue inévitablement, la fille - version édulcorée, universelle, idéelle des filles - étant une abstraction, un fantasme calqué sur les «besoins» et les «intérêts» des hommes (NML, p. 271). L'illusion ne dure qu'un temps; le temps, pour l'homme, de "couche[r] avec une image», «une revenante», «un fantôme de rechange» («c'est ce qu'il préfère», précise la narratrice; GP, p. 86) qui éclipse l'être de chair dissimulé, voire étouffé derrière. La narration ultérieure, laquelle octroie à Cora Corail un certain recul par rapport aux événements racontés et, conséquemment, une certaine clairvoyance qui va de pair avec la «conscience de genre ${ }^{44}$ » désormais acquise permet, par ailleurs, de mettre au jour le décalage existant entre la fille-fantasme (le masque) et la fille réelle. Si la fille-fantasme se caractérise avant tout par «ses postures vivantes, ses maquillages imperturbables, la légèreté inscrite sur son visage, toujours serein, rarement éploré» (GP, p. 83), la fille réelle n'est que «l'ombre de [s] on ombre», elle a «le ventre creux», «les idées noires» (GP, p. 54-55) et est «ravagée par l'insomnie» (GP, p. 134).

Des deux narratrices de l'œuvre, Cora Corail est la première à se rendre à l'évidence ( J'essaie aussi ce visage. Il me tombe du crâne. Ça ne tient pas, Bertha»; GP, p. 83) et à se départir de la «surface jetable» $(G P$, p. 106) que représente le masque de la féminité normative. Si elle parvient de ce fait à se libérer, momentanément du moins, du poids que celle-ci fait peser sur les filles ${ }^{45}$, elle n'en est pas moins assaillie de doutes: «Y a-t-il une vie après le lait démaquillant? [...] Y a-t-il un être dans le bustier de satin, dans le regard

appelée. J'ai décrit une soirée type, de laquelle, ce soir, je me suis passée. Voilà ce qui aurait toujours bien pu, une fois de plus, arriver.» (GP, p. 85 ; je souligne)

43. Ainsi s'adresse-t-elle à Léa: «Toi aussi, tu crois encore aux mythes et au hasard, aux héros délicats, aux brutes compréhensives et aux gorilles qui préparent le café du matin?» (GP, p. 79; je souligne)

44. Eleni Varikas, La révolte des dames, ouvr. cité, p. 10-11; 13-14.

45. Avant de laisser tomber le masque de la féminité, Cora Corail confie à Léa être «très lourde dans les bras des hommes et que ça ne va pas.» (GP, p. 93) 
froid comme une autopsie, dans cette femme qui nage dans la crème et soigne ses effets, se désire portrait-robot, extrême survivante, les canons de la beauté pointés sur ses seins, son faciès, partout, dans son dos? Y a-t-il une vie pour les poches de patates?» (GP, p. 88-89) Les questions que soulève l'œuvre de Monette sont cruciales: qui est la fille derrière le masque? Quelle vie est réservée à celle qui le retire? Est-il seulement possible d'exister sans le masque ${ }^{46}$ ? Pas sans un réaménagement considérable des termes qui définissent le genre et, plus particulièrement, le script hétérosexuel, nous indique le récit que tient Cora Corail de «l'année où [elle] devin[t] laide»: "arriva ce qui devait arriver: l'épouvante. Non, l'angoisse, plutôt; c'est moins hyperactif, moins grandiose, plus proche de cette réalité brisée qui nous sert d'impuissance.» (GP, p. 95) L'expérience de l'angoisse que traverse Cora Corail après avoir abdiqué devant les exigences du modèle hétéronormatif est en partie rendue depuis la perspective de Léa qui, elle, n'y a pas encore renoncé:

Je me souviens de ce rendez-vous, de cette pagaille. C'était le mois de novembre suivant le mars de notre alliance, vers la toute fin du mois. Non seulement elle [Cora] avait perdu le mordant de sa folie, le génie de son insolence, mais elle n'était plus que le cadavre des violences qu'elle s'était poussée à vivre. Introvertie, tremblotante, vulnérable jusqu'à la défaite prévue d'avance. Insupportable. (GP, p. 104)

46. Dans son article portant sur l'œuvre de Vanessa Beecroft et celle de Nelly Arcan, Martine Delvaux pose des questions similaires à propos de la femme en tant qu'image. Elle conclut que l'artiste et l'écrivaine «font chuter les femmes [...], et ce qu'elles provoquent, c'est la chute de l'image. Non pas dans un geste où l'image serait un vêtement dont on pourrait se dépouiller pour arriver au vrai; mais au sens, plutôt, où l'image apparaîtrait comme la vie elle-même. Il n'y a rien au-delà ou en-deça de l'image. Ainsi, il est vrai, la femme est image.» ( EÉcriture et nudité. Les femmes de Nelly Arcan et de Vanessa Beecroft», Tangence, $\mathrm{n}^{\circ} 103$ [Polygraphies du corps dans le roman de femme contemporain, dir. Andrea Oberhuber], 2013, p. 91.) C'est bien ce que la culture, élaborée à partir du male gaze, nous dit, et c'est également ce que confirme Cora Corail en faisant allusion à son dernier amant: "Il aimait les masques que je portais pour lui. Les masques ne tenant jamais bien longtemps, je n'étais pas une femme, je n'étais plus une femme selon lui.» (GP, p. 86) Cette réduction des filles et des femmes à l'image est bien ce qui engendre, chez Cora Corail, l'impression de disparaître une fois le masque de la féminité normative retiré. Car le male gaze ne voit pas ce qui se cache derrière le masque. Pour refaire surface autrement, les filles doivent se tourner vers un autre regard - celui de leurs sœurs, en l'occurrence. 
Aussi, bien que le récit demeure imprécis à ce sujet, il semble que ce soit cette entreprise de résistance aux normes en vigueur qui soit responsable de la «maladie» qui afflige Cora Corail, décrite, par Léa, comme « une maladie du malheur, une maladie de digestion du malheur qui avait tout fait pour l'emporter. Une maladie pour mourir, très ordinaire, et comme telle, sans doute liée à l'ennui que procure la destruction» (GP, p. 108). Le séjour qu'effectuera éventuellement Cora Corail à l'hôpital apparaît, par ailleurs, comme l'aboutissement ultime de cette «maladie» qui, selon la narratrice, «fait partie de [l'] éducation [des filles] ${ }^{47} »(G P$, p. 152). Comme l'ont montré de nombreuses féministes, les filles apprennent très tôt à se penser à travers des schèmes symboliques élaborés en fonction du sujet référentiel masculin. Elles intériorisent le rôle qui leur est prescrit, soit celui de refléter le désir des hommes ${ }^{48}$. Ce processus de colonisation de la pensée et de l'imaginaire instaure des hiatus dans la pensée, desquels découle une conscience potentiellement divisée de la réalité. Selon Nicole-Claude Mathieu, les hommes formeraient un «véritable écran, dans le double sens d'objet interposé dans [1] a conscience [des femmes], et de surface opaque d'où [leur] est renvoyée une sorte de logique de la contradiction dans la conduite de [leur] propre vie ${ }^{49}$ ». Cette contradiction entre sentiment de soi et rôle imposé trouve écho dans le discours de Cora Corail:

Une autre de nos ${ }^{50}$ manies catastrophiques, c'est la contradiction. Notre esprit tortueux, comme saturé d'analyses émotives, psychologiques, affectives et culpabilisatrices, notre esprit abusé par ses millions de contradictions, débouche sur quoi, tu penses, Gemma? Sur l'hystérie et ses dérivés multiples: une tendresse excessive comme une rage démesurée, une haine incoercible

47. Il est possible de voir, dans la description que Léa fait de Cora Corail, les signes de l'anorexie, qui peut être lue, sur le plan symbolique, comme façon de pallier la surreprésentation du corps des filles au détriment de leur intellect. À ce sujet, voir Donna J. Haraway, Modest_Witness@Second_Millenium.FemaleMan -_Meets_OncoMouse ${ }^{\mathrm{TM}}$, New York/Londres, Routledge, 1997, p. 32.

48. Sur ce point, voir Anne-Marie Dardigna, Les châteaux d'Éros ou les infortunes du sexe des femmes, Paris, François Maspero, 1980, p. 15.

49. Nicole-Claude Mathieu, "Quand céder n’est pas consentir», art. cité, p. 153; l'autrice souligne.

50. Cora Corail fait ici référence au groupe des filles, dans son ensemble. 
comme une sincérité larmoyante, une méchanceté vindicative comme un désir de partage. (GP, p. 152) $)^{51}$

Le système patriarcal en place produit ainsi des sujets féminins constamment tiraillés entre l'obéissance aux normes édictées - la conformité au modèle de féminité instauré et valorisé par le système étant récompensée par diverses gratifications (NML, p. 273) et assurant une certaine reconnaissance, nécessaire à l'acquisition d'un statut légitime dans la sphère publique - et la révolte, laquelle expose les filles qui s'y adonnent au mépris, aux représailles, voire à la folie ( «Les pires d'entre nous sont déchirées. Les pires d'entre nous sont folles», s'indigne Cora Corail; GP, p. 152). Le caractère irréconciliable de ces deux postures participe également de la désolidarisation des filles, dans la mesure où celles qui continuent de se conformer sont potentiellement perçues, par leurs sœurs, comme complices de la loi des hommes, tandis que celles qui décident de se rebeller sont, elles, tenues responsables de leur propre malheur, de leur propre destruction, et même de celle des autres. Il faut bien voir, à cet effet, que le modèle hétérosexuel romantique, en tant que performance scriptée, requiert, afin que soit maintenue l'illusion de la réalité, que toutes tiennent leur rôle. Imaginons une scène de théâtre: il suffit qu'une seule comédienne retire son masque pour révéler le fait que chacune en portait un depuis le début de la représentation - alors même que le propre de la mascarade est de "produire sur un mode performatif une ontologie sexuelle, un paraître qui réussit à passer pour un "être" 52 ». Qu'une seule l'enlève, et toute l'attention est portée sur le masque lui-même en tant qu'artifice, et sur celles qui le portent encore. Qu'une seule l'enlève, et toutes seront taxées d'hypocrisie, déclarées superficielles, vaines: des simulatrices. Voilà qui peut expliquer le différend qui s'installe progressivement entre Léa et Cora Corail ${ }^{53}$, alors que cette dernière tente de la convaincre de la suivre dans cette nécessaire, bien que douloureuse, entreprise de décolonisation du corps et de l'imaginaire:

Allez, Natacha! Permets-toi une petite crise de Belle au Fer Brûlant! Révèle-toi. Montre tes belles dents et tes miroirs au

51. On peut lire, dans le discours de Cora Corail, l'intériorisation des clichés patriarcaux, qu'elle reprend afin de mieux les exposer et, partant, de les dénoncer.

52. Judith Butler, Trouble dans le genre, ouvr. cité, p. 131.

53. «Je suis une créature isolée, un monstre. Tu es seule et en beauté. Nous n'avons rien à faire ensemble», se désole Cora Corail (GP, p. 114). 
rebord sec. Aplatis-moi de ta splendeur, empoisonne-moi des inflexions congelées de ta voix céleste, au-dessus du commun des mortels, au-delà des franchises de la médiocrité, et pour masquer jusqu'à l'injure qui t'atteint tout de même un peu, parle-moi encore des nouveaux produits de beauté qui jalonnent tes espérances de vie.

Tu me maudis? Tu pleures? Tu t'attends à quoi au juste des filles comme toi? (GP, p. 96-97)

Cette requête ne reçoit pas, toutefois, la réponse escomptée. Aussi, la première réaction de Léa est de se sentir irritée et de rejeter, en bloc, ce que lui propose Cora Corail. Ainsi s'exprime-t-elle après l'écoute de la troisième cassette: «Elle me fait pitié; c'est sans compassion. Il n'y a plus d'amitié, je l'ai découvert; c'est brûlé. Son défaitisme, son absence de beauté durable, c'est trop moche pour mes moyens. Et là, c'est assez, ça va plus loin, ça va trop loin, c'est fini, Cora. C'est fini, ma douce ordure.» (GP, p. 140) Il faudra attendre l'écoute de la quatrième cassette, celle où le rêve d'une communauté solidaire se fait le plus explicite, pour venir à bout des résistances de Léa, et que celle-ci réponde à l'appel de son amie.

\section{La constellation des filles: vers un nouveau symbolisme au féminin}

Sortie, de son propre gré, du marché hétérosexuel, Cora Corail perd progressivement «le sentiment de sa [propre] réalité ${ }^{54} »$. L'état de déréliction dans lequel se trouve plongée la narratrice ${ }^{55}$ semble redevable de la croyance, induite par le script romantique traditionnel, que seule la reconnaissance octroyée par un homme peut «fourni[r] l'accès au monde qui [...] était fermé autrement [aux filles $]^{56} »$. Or, ce n'est plus en tant qu'objet soumis à l'appréciation de l'autre masculin que Cora Corail souhaite désormais faire partie du monde et accéder à une quelconque forme de pouvoir. Pour mettre fin à cette dépendance aliénante au regard des hommes, Cora Corail en appelle à un changement des règles qui gouvernent

54. Jessica Benjamin, Les liens de l'amour, ouvr. cité, p. 24.

55. Cette impression d'assister à sa propre disparition est confirmée par une demande qu'elle adresse à Léa: «Est-ce qu’on pourrait se voir bientôt? Je voudrais être sûre. [...] Je voudrais simplement que tu me dises si tu me vois.» (GP, p. 127)

56. Jessica Benjamin, Les liens de l’amour, ouvr. cité, p. 121. 
les rapports entre les sexes, mais aussi, peut-être surtout, les rapports entre les filles.

Afin de renverser le modèle prédominant, qui repose sur l'abnégation des filles, c'est-à-dire sur le renoncement de leur propre intérêt au profit de la classe masculine dominante, il est nécessaire d'élaborer un nouveau modèle de sororité, fondé sur une «solidarité politique» laquelle, selon bell hooks, doit se penser en fonction du potentiel agentif des filles et des femmes et non simplement en termes de victimisation ${ }^{57}$. Cette idée se fait jour, dans le discours de Cora Corail, tout d'abord de façon presque inopinée, d'une façon qui n'est réfléchie, comprise par la locutrice qu'au moment où elle l'énonce: «J'ai la manie de parler à la première personne du pluriel. [...] comme si je voulais nous forcer la main pour que nous puissions saisir, ensemble et floues, quelques miettes d'une quête honorable et solidaire.» (GP, p. 153-154; je souligne) Le «nous» apparaît ainsi s'imposer de lui-même, comme une évidence, suggérant par là que la solidarité féminine représente bien l'unique remède à la «maladie du malheur » qui ronge les filles (GP, p. 108). Puis, le désir de revenir à une forme d'amitié vraie, inscrite dans la durée, prend la forme d'une invitation claire:

Écoute, Esméralda, c'est pas fini. J'aimerais qu'on se retrouve, toujours dignement, jusqu'à nos 88 ans. Deux cérébrales comme nous, continuant à brasser la planète avec nos idéaux tremblotants et notre terrible attachement, envers comme à l'envers des princes amnésiques et troublants. Ramassant les bribes saines de nos restants de cerveaux et bravant l'histoire de nos mots poussiéreux. Nous pourrions d'ailleurs être deux, cinq ou dix. Les vieilles rescapées de l'an 2048. Vieilles sorcières faisant les belles, scrutant chaque moment des années passion. Discourant sur des siècles de

57. C'est ce modèle qui, selon bell hooks, a conduit les féministes blanches bourgeoises à rejeter les femmes noires des mouvements féministes: «La complicité entre victimes a créé une situation dans laquelle les femmes confiantes qui s'affirmaient d'elles-mêmes étaient souvent perçues comme n'ayant pas leur place dans le mouvement féministe. C'est cette logique qui a mené les militantes blanches (avec les hommes noirs) à suggérer que les femmes noires étaient si "fortes" qu'elles n'avaient pas besoin d'être actives dans le mouvement féministe.» («Sisterhood: Political Solidarity between Women», art. cité, p. 128; je traduis.) «Bonding as victims created a situation in which assertive, self-affirming women were often seen as having no place in feminist movement. It was this logic that led white women activists (along with black men) to suggest that black women were so "strong" they did not need to be active in feminist movement." 
guerre et d'espoir, impitoyables sœurettes ne s'épargnant rien des détails du voyage épouvantable, presque alors, enfin, détourné de son illusion originelle. (GP, p. 156)

C'est à une relation d'amitié fondée sur la volonté toujours renouvelée de déconstruire ce qui empêche les filles de se constituer comme sujets (ici désigné par la référence à "des siècles de guerre») que Cora Corail convie Léa, et les «cinq ou dix» autres qui voudront bien les rejoindre. Une amitié engagée, donc, à réécrire l'histoire des filles; une amitié née d'une expérience partagée de la domination, et culminant dans la réappropriation d'une parole dénonciatrice: «Si mon corps me coupe la parole à tout bout de champ, [prévient Cora,] je finirai par hausser le ton. Je veux parler, Deborah. Je vais bientôt crier, est-ce que tu m'entends? Tu vis la même chose que moi, n'est-ce pas? Dis-moi que tu m'écoutes encore, que tu t'écouteras.» (GP, p. 55)

Afin d'arriver à ce renouveau des rapports entre filles, il importe de procéder à la réhabilitation de certaines valeurs, dont l'entraide, la sollicitude, la douceur et la réciprocité ${ }^{58}$, historiquement associées au féminin et, par le fait même, discréditées (au profit, notamment, de la valorisation de l'autosuffisance - illusion soutenue par l'idéologie néolibérale ${ }^{59}$ — et du détachement des affects $\left.{ }^{60}\right)$. Le récit illustre bien la nécessité de ces valeurs, dont la mise en pratique, par les filles, contribue littéralement à leur sauver la vie le soir de leur première rencontre, alors qu'un chauffeur de taxi tente de les écraser avec sa voiture. Devant la furie du chauffard, elles prennent la fuite ensemble, propulsées par «la vie [qui] hurl[e]» en elles (GP, p. 30). Si l'une trébuche, l'autre l'aide à se relever (GP, p. 30) et c'est

58. Ces valeurs renvoient par ailleurs explicitement à celles qu'énumère Élaine Audet dans l'avant-propos de son ouvrage Le cour pensant. Courtepointe de l'amitié entre femmes (ouvr. cité, p. 9-20), faisant référence aux principes fondateurs des amitiés féminines. À ce propos, il importe de souligner que ce travail politique de réhabilitation des valeurs dites «féminines» est largement attribuable aux penseuses et aux penseurs de l'éthique du care. Voir, entre autres, Sophie Bourgault et Julie Perreault (dir.), Le care. Éthique féministe actuelle, Montréal, Les éditions du remue-ménage, 2015.

59. À ce sujet, voir Frédéric Lordon, La société des affects. Pour un structuralisme des passions, Paris, Seuil, coll. «L'ordre philosophique», 2013.

60. Voir Patricia Paperman et Pascale Molinier, «Préface», Contre l’indifférence des privilégiés. À quoi sert le care, éd. Patricia Paperman et Pascale Molinier, Paris, Payot, 2013, p. 21. 
main dans la main qu'elles parviennent à fuir les lieux et à échapper à leur «bourreau» (GP, p. 31). Ainsi raconte Léa: «le visage à deux doigts du massacre, la noirceur des roues à portée de la joue, nous avons senti le caoutchouc brûlant, la frénésie, la mort, j'étais là aussi, j'ai crié avec elle; c'est cela: nous avons hurlé. [...] j'ai pris mon amie par la main, l'ai entraînée plus loin, encore trop près, très vite.» (GP, p. 31) Cette scène particulière, dans laquelle se déploie de façon spectaculaire la violence patriarcale - tout comme la résistance féminine à cette violence - , semble contenir l'essentiel du roman, le scénario qui se répètera en boucle jusqu'à la toute fin, et qui repose sur l'opposition farouche des hommes à la complicité des filles.

La suite de l'œuvre peut ainsi être lue comme le souhait exprimé par Cora Corail de revenir à cette complicité première, telle qu'elle se déployait entre les filles avant d'être mise en péril par les impératifs reliés au script romantique. Les enregistrements envoyés par Cora Corail à Léa contiennent, à cet effet, le fruit d'une longue réflexion partagée avec l'amie perdue dans l'espoir qu'elles retournent à ce point de départ où, ensemble, elles s'employaient spontanément à prendre soin l'une de l'autre. À titre d'exemple, les filles avaient l'habitude, aux premiers temps de leur amitié, de s'assurer que l'autre se nourrissait bien, en opposition, assurément, aux diktats de la beauté qui normalisent, voire érotisent la maigreur des filles et les affament: «Léa, je te le demande encore: as-tu mangé aujourd'hui? » (GP, p. 55) De même, le soir de leur rencontre au bar le Snob, Léa, après s'être rendue compte que Cora Corail était «fauchée», n'avait pas hésité à lui «pay[er] un café», tout en s'inquiétant que celle-ci soit «pieds nus dans ses souliers» en plein mois de mars: "C'est pas croyable. Tu vas geler!» (GP, p. 25-26) Enfin, la bienveillance de Léa à son endroit est exprimée à travers sa volonté de l'aider à trouver un nouveau logement afin de la sortir de la "piaule» malfamée où elle habite - laissant ainsi voir son désir de la voir s'épanouir: "Peutêtre que je me laissais aller comme dans un rêve, pour la sortir du cauchemar, la séparer des crétins et des avares, lui régler son compte, qu'elle en finisse de sous-exister dans ce pensionnat de paumés.» (GP, p. 65)

Comment éviter que cette relation privilégiée ne soit à nouveau menacée par la rivalité qu'instaurent les scripts hétéronormatifs? C'est la question à laquelle le discours de Cora Corail semble vouloir donner une réponse. Il faut, certes, passer à l'action, comme le laisse 
entendre ce passage où la narratrice élabore des projets pour que les injustices vécues sur le plan intime soient dénoncées sur la place publique: «Au mieux, nous pourrions simuler une évasion, inviter des journalistes, finir dans les journaux. Par médiateur interposé, nous exigerions de parler à la radio de tout ce qui nous fait mal.» (GP, p. 94) Mais il faut aussi que les filles se donnent le pouvoir de se reconnaître entre elles, et cela nécessite, comme l'avançait Irigaray, la création d'un nouveau symbolisme au féminin.

L’image proposée par Monette, à travers le personnage de Cora Corail, afin de penser un nouveau modèle de relations entre filles, est celle de la constellation. À ce sujet, il faut voir que le texte est parsemé de métaphores renvoyant à l'espace céleste. Lors de leur première rencontre, la narratrice «abord[e] [Léa] en devinant du premier coup [s] on signe astrologique» $(G P$, p. 21$)$. De plus, aux yeux de Léa, les deux filles sont des «jumelles astrales» (GP, p. 108). Cora Corail, de son côté, voudrait avoir "un appart bien à elle, accroché au ciel» (GP, p. 61), et lorsqu'elle réfléchit aux rapports entre les hommes et les femmes, elle déclare qu' «Orion et Cassiopée ${ }^{61}$ ne partageront jamais le même hémisphère» $(G P, \mathrm{p} .133)$.

Cette constellation prend forme notamment par le choix que fait Cora Corail de s'adresser à Léa en lui attribuant chaque fois un nouveau prénom, mais toujours avec une finale en «a»: Eva, Cynthia, Francesca, Tania, Ursula, etc. Le premier réflexe de Léa est d'en être offusquée: «Elle me traite de tous les noms, elle veut oublier celui que je porte. [...] Comme si j'étais toutes les filles» ( $G P$, p. 20). Certes, il est possible d'interpréter l'usage de nombreux différents prénoms pour interpeler une seule personne comme une façon de souligner - en même temps que de critiquer - l'interchangeabilité des filles au regard de l'idéologie patriarcale. Monette va toutefois plus loin que cela, en redonnant ultimement, à chacune, son identité propre, récusant, de ce fait, l'adage misogyne voulant que les filles soient «toutes les mêmes»:

Je m'excuse pour tantôt, Rita. Je suis allée trop loin, les mots ont dépassé ma pensée, j'ai exagéré. Au sujet des filles, j'ai trop

61. La référence à ces deux constellations, qui sont aussi des figures de la mythologie grecque, n'est pas fortuite. Orion, chasseur réputé pour son impétuosité, condense les traits de la masculinité hégémonique décriée par Cora Corail. Quant à Cassiopée, elle est celle qui déclenche la colère des nymphes en affirmant que sa fille, Andromède, est plus belle que ces dernières. 
généralisé, je me suis emportée, j'ai dit n'importe quoi. Je ne pense pas qu'on soit toutes comme ça. Non, sincèrement, je ne le crois pas. À vrai dire, Noëlla, en te disant tantôt ce que j'ai beuglé si souvent à part moi, je ne voulais pas parler de toutes les filles. Non. (GP, p. 82)

Ce retour à soi, cet acte de parler d'abord pour soi, ne bafoue pas pour autant l'expression d'une solidarité; au contraire. Parler au nom de toutes, sans distinction, on le sait, a contribué à perpétuer, au sein même des mouvements féministes, les schèmes de domination racistes, classistes, âgistes, capacitistes et cissexistes, entre autres. La formation d'une communauté solidaire, dans Le goudron et les plumes, passe donc avant tout par la reconnaissance de la singularité de chacune, en commençant par celle de l'amie la plus proche: «Mais Léa, que je te nomme par ton nom cette fois, cent fois, car c'est le bon et il n'y a pas d'autres toi.» (GP, p. 154) Le fait d'énoncer autant de noms de filles représente, à cet égard, un geste symbolique, que l'on peut percevoir - pour faire écho à l'image de la constellation proposée par Monette - comme une volonté de rapatrier les étoiles solitaires, de les faire advenir comme sujets et, en les nommant ainsi une par une, à les faire briller «comme de fluorescentes citées dans la nuit patriarcale ${ }^{62} »$.

Comme le souligne Françoise Collin, «[la culture patriarcale] n'a pas vidé le ciel des femmes, elle l'a au contraire rempli, et bouché, à l'aide d'une identité ou pseudo identité qui les dispensait de s'inventer» (NML, p. 272). La constellation imaginée par Hélène Monette est une façon d'ouvrir le ciel des filles, laissant le loisir à chacune d'elles - à partir à la fois de son expérience singulière et en dialogue avec les autres - de dessiner de nouvelles formes, de nouvelles configurations stellaires, élaborant ainsi une nouvelle

62. Nicole Brossard, La lettre aérienne, Montréal, Les éditions du remue-ménage, 2009, p. 82. La proposition de Monette rejoint, en cela, celle qu'énonce France Théoret dans un entretien avec Louise Dupré en 1986. Comme le rapporte Isabelle Boisclair dans sa présentation de l'écrivaine dans le Dictionnaire des intellectuel-les au Québec, "[t] el qu’elle [Théoret] le pense, le "je” "préside au nous" (Vertiges, 1979): "il ne prend pas la parole au nom de toutes les femmes, il ne fonde pas le collectif, mais appelle une collection de voix qui se joignent les unes aux autres pour créer une polyphonie, un effet de profondeur." (Dupré, 1986).» («France Théoret», dans Yvan Lamonde et coll. [dir.], Dictionnaire des intellectuel-les au Québec, Montréal, Presses de l'Université de Montréal, coll. «Corpus», 2017, p. 304; l'autrice souligne.) 
mythologie au féminin. L'histoire de Cora Corail et de Léa en est une d'amitié vive, mais c'est aussi l'histoire d'une fragilité; celle qui affecte potentiellement les relations entre filles, dans un monde gouverné par la loi des hommes. Comme le souligne Cora Corail, il n'y a «[a]ucune amie qui règne sur l'amitié» (GP, p. 93). Aucun ordre - outre le nôtre, qu'il nous revient de construire et de préserver - ne veille à la pérennité des amitiés entre filles, ni ne protège nos alliances, contrairement à l'amour romantique. "La veilleuse» $(G P$, p. 162) féministe est, à cet égard, indispensable pour maintenir «cette foi complice, cette christ d'ardeur qui nous rattache les unes aux autres. Les unes aux autres.» (GP, p. 165) 